\title{
Shunt Technology in Pediatric Neurosurgery: Current options and Scientific Evidence
}

\author{
Luciano Furlanetti ${ }^{1}$, Matheus Fernando Manzolli Ballestero ${ }^{2}$, Ricardo Santos de Oliveira ${ }^{3}$
}

Received: 09 April 2020 / Published: 18 June 2020

\begin{abstract}
Introduction: Hydrocephalus is the most common neurosurgical condition among children worldwide and shunt diversion of the cerebral-spinal fluid (CSF) has been widely available. The aim of the present study was to perform a systematic review on types and models of shunt devices available and critically evaluate scientific evidence in favor of the usage of specific technologies.

Methods: Searches of the PubMed database were performed for relevant articles published until March 2020. Appropriate keywords were used to identify all studies, including: "shunt", "technology", "hydrocephalus", "children", "pediatric". The indications and scientific evidence in favor of the usage of specific shunt technology for the treatment of hydrocephalus according to age, underlying pathology and other features of specific patients were discussed under the light of the current literature.

Results: A total of 178 peer-reviewed papers were found. Out of them, only 21 matched our inclusion criteria and were finally selected. The papers were reviewed and described technology discussed below. A summary of the main types of shunts commercially available worldwide, including their respective working mechanism and construction was also included.

Conclusion: Shunt technology has evolved during the last decades and continues to be intensively developed. Despite the huge arsenal of different types of shunt systems currently available on the market, the treatment of hydrocephalus remains challenging. Investment in research, education and training, as well as prospective randomized multicentric controlled trials evaluating the role of specific valves in the treatment of individual group of hydrocephalic pediatric patients are urgently needed.
\end{abstract}

Key words: hydrocephalus, shunt-devices, technology, pediatric neurosurgery

\footnotetext{
1'Department of Neurosurgery, King's College Hospital NHS Foundation Trust, Denmark Hill, London, UK

${ }^{2}$ Department of Medicine, Federal University of São Carlos, São Carlos, Brazil
}

\footnotetext{
${ }^{3}$ Division of Neurosurgery, Department of Surgery and Anatomy, Ribeirao Preto Medical School, University of Sao Paulo, Brazil
}

To whom correspondence should be addressed: Luciano Lopes Furlanetti, MD, PhD [E-mail:furlanettil@gmail.com]

\author{
Introduction \\ "The history of evolution of ventricular shunting is largely a \\ history to prevent the complications of shunting." R. MacLaurin
}

Hydrocephalus is the most common neurosurgical condition among children worldwide. Its surgical management by means of shunt diversion of the cerebral-spinal fluid (CSF) has been widely available for a long time and is considered a highly effective solution to most cases. Despite of the advent, development and popularization of endoscopic techniques in the treatment of hydrocephalus, shunt technology experienced unparallel improvement during the last decades. Since the first implantable shunt device described by Nulsen and Spitz in the late 40's,(1) using a stainless-steel ball-valve system, shunt systems evolved to distal slit valves, proximal slit, diaphragm, anti-siphon systems, gravitational and more recently the electromagnetic adjustable mechanisms, to mention a few examples(2). Along with the development of valve mechanisms, the development of silicone tubing and antibioticimpregnated systems contributed enormously to the quality of the shunt devices(3). Until recently, only limited options of shunt devices were available. Advances during the last decades led neurosurgeons to face the complex challenge of having to select the ideal shunt system that is appropriate for an individual patient based on several variables, such as age, underlying pathology, daily activity and costs. Currently, a myriad of companies offers a variety of shunt systems worldwide. If in the past the question was whether to implant a "low-, medium or highpressure" device, nowadays this has become more complex, so that one of the main factors limiting the choice is the cost. However, one has to bear in mind that the latest technology may not always be the best option. Factors such as age of the patient, health conditions, baseline disease, physical activity, insurance coverage, commercial availability of the 
shunt device and postoperative care/maintenance, not no mention the individual professional experience, should be taken into account. On one hand, last generation adjustable shunt devices coupled with antigravitational mechanisms offer the possibility of fine follow-up titration according to individual patient's needs, however these are extremely costly and demand experience. On the other hand, simple differential pressure valves are robust, widely available and accessible even in low-income countries, nevertheless they may be associated with higher incidence of postoperative complications, such as siphoning, subdural hematoma and other wellknown complications of overdrainage. The aim of this study was to perform a systematic review of the literature on types and models of shunt devices available and critically evaluate the scientific evidences and recommendations in favor of specific technologies for individual patients.

\section{Methods}

A literature search was performed to identify shunt technologies for treatment of hydrocephalus. Searches of the PubMed database were performed for relevant articles published from until March 2020. Appropriate keywords and MeSH terms were used to identify all studies, including: "shunt", "technology", "hydrocephalus", "children", "pediatric". The reference lists of papers were also manually searched to identify additional data sources. Included papers described the use of new technology in vivo. Experimental, in vitro and papers on animal research were excluded. The findings, indications and scientific evidence in favor of the usage of specific shunt technology for the treatment of hydrocephalus according to age, underlying pathology and other features of specific patients were discussed under the light of the current literature.

\section{Results}

A total of 178 peer-reviewed papers were found. Out of them, only 21 matched our inclusion criteria and were finally selected. The literature search process is presented in the form of a flowchart in Figure 1. The papers were reviewed and described technology discussed below. Table 1 summarizes the diverse main types of shunts commercially available worldwide and in Brazil, including their respective working mechanism and construction.

\section{Discussion}

\section{CSF physics and hydrocephalus}

Hydrocephalus is a common problem in the clinical practice of every neurosurgeon. Having decided that a hydrocephalic patient needs a shunt, the next common question even for the more experienced

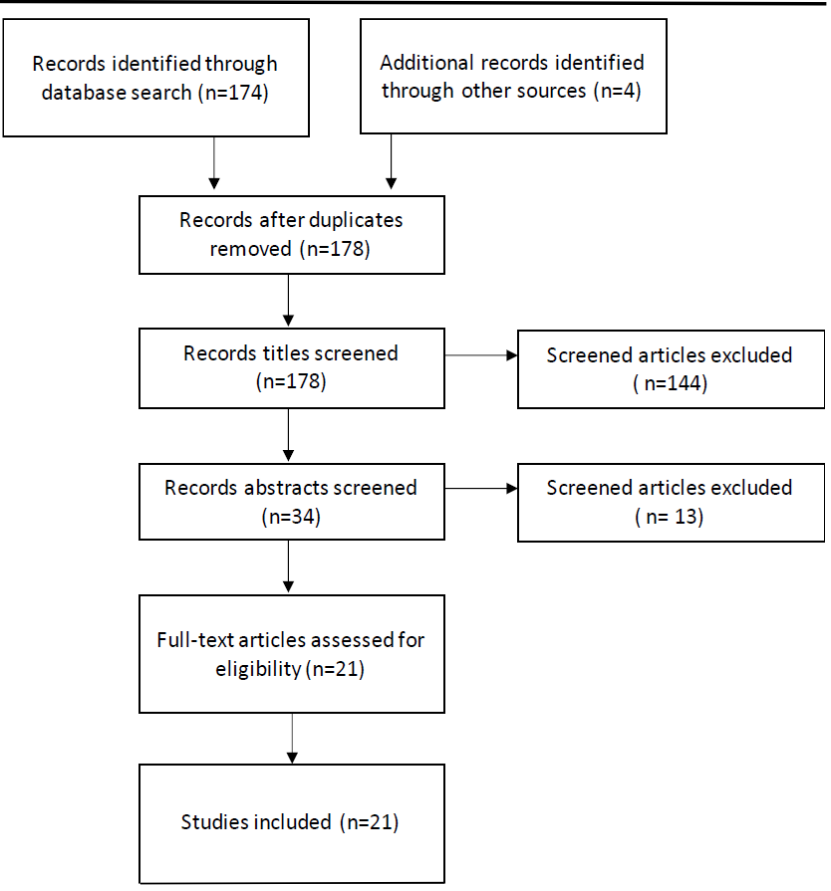

Figure 1 - Flowchart depicting the process for review of the literature

neurosurgeon nowadays is which shunt to use. Independently of the type of shunt system employed, the flow rate $(Q)$ is in direct relation with the driving pressure $(P)$ and in indirect relation with the resistance $(R)$, represented by $Q=P R$. Factors influencing the resistance within the tubing $(\mathrm{Rt})$ are the length $(\mathrm{L})$ and inner diameter of the distal catheter and the viscosity (n) of the CSF $\left(R t=8 n L / \pi r^{\wedge} 4\right)$. Therefore, the pressure gradient driving the flow in a shunt system is determined by $\Delta P=(I V P+\rho g h)-(O P V+R t+D C P)$, where IVP stands for "intraventricular pressure", $\rho g h$ for "hydrostatic pressure", OPV for "opening pressure of the valve" and DCP for the "distal cavity pressure". As in the horizontal position, the IVP is positive $(+10 \pm 5$ $\mathrm{mmHg}$ ), whereas in standing/sitting position, the IVP becomes slightly negative $(-5 \pm 5 \mathrm{mmHg})$ and the hydrostatic pressure has a major effect on flow. Therefore, the sum of the opening pressure of the shunt system, resistance of the tubing and the pressure within the distal cavity play together an important role to avoid overdrainage in the upright position (4). (Figure 2). However, the exact flow in a shunt system is difficult to predict, because of the several factors changing shunt resistance and driving pressure along the day, so that it can vary by a factor of 200 times in few hours, depending of activity and position of the body $(4,5)$.

\section{Technological developments}

First attempts of a ventriculoperitoneal shunt by Kausch and later by Cone, Scott and JacksonSnodgrass were historically reported in Handbuch der Neurochirurgie, with mean mortality rate ranging from 5 to $38 \%(6)$. With the advance of neurosurgical 
Table 1 - Types of shunts commercially available worldwide and in Brazil, including their respective working mechanism and construction. The devices are sorted by manufacturer

\begin{tabular}{|c|c|c|c|c|}
\hline Shunt Name & Manufacturer & Functionality & Construction & Brazi \\
\hline PaediGAV & Aesculap-Miethke & $\mathrm{G}$ & ball on spring & yes \\
\hline ProSA + miniNAV & Aesculap-Miethke & $A D J+G$ & ball on spring & no \\
\hline ProGAV & Aesculap-Miethke & $A D J+G$ & ball on spring & yes \\
\hline M Blue & Aesculap-Miethke & $A D J+G$ & ball on spring & no \\
\hline Shunt reservoir & Aesculap-Miethke & ICP telemetry & $\mathrm{N} / \mathrm{A}$ & no \\
\hline Dual Switch & Aesculap-Miethke & G & diaphragm \& spring & no \\
\hline HLL Life Care Limited & Ceredrain & $\mathrm{DP}$ & diaphragm & no \\
\hline Chhabra & Chhabra & $\mathrm{DP}+\mathrm{ASD}$ & slit and spring & no \\
\hline Hakim Precision Valve & Codman & $\mathrm{DP}$ & ball on spring & no \\
\hline Hakim Adjustable & Codman & ADJ & ball on spring & yes \\
\hline Unishunt & Codman & $\mathrm{DP}$ & distal slit & no \\
\hline Accu-Flo & Codman & $\mathrm{DP}$ & silicone membrane & no \\
\hline Holter Valve & Codman & $\mathrm{DP}$ & proximal slit & no \\
\hline Sphera duo & HPBio & $\mathrm{DP}$ & ball on spring & yes \\
\hline Sphera pro & HPBio & $A D J+G$ & ball on spring & yes \\
\hline Omnishunt & Integra & $\mathrm{DP}$ & ball on spring & no \\
\hline Hakim Valve & Integra & $\mathrm{DP}$ & ball on spring & no \\
\hline In-Line Valve & Integra & $\mathrm{DP}$ & mitre & no \\
\hline Orbis Sigma Valve & Integra & Flow-regulating & diaphragm & yes \\
\hline Low Profile Valve & Integra & $\mathrm{DP}+\mathrm{ASD}$ & silicone membrane & no \\
\hline $\begin{array}{l}\text { Pudenz Flushing Valve } \\
\text { w/ ASD }\end{array}$ & Integra & $\mathrm{DP}+\mathrm{ASD}$ & silicone membrane & no \\
\hline Strata & Medtronic PS Medical & $A D J+A S D$ & ball on spring & yes \\
\hline Strata NSC & Medtronic PS Medical & ADJ & ball on spring & yes \\
\hline Strata 2.0 & Medtronic PS Medical & $A D J+A S D$ & ball on spring & yes \\
\hline CSF Lumbop.Shunt & Medtronic PS Medical & $\mathrm{DP}$ & distal slit & yes \\
\hline Delta Valve & Medtronic PS Medical & $\mathrm{DP}+\mathrm{ASD}$ & silicone membrane & no \\
\hline CSF Flow Control V. & Medtronic PS Medical & $\mathrm{DP}$ & silicone membrane & no \\
\hline Novus & Natus & $\mathrm{DP}+\mathrm{G}$ & silicone membrane & no \\
\hline Contour-Flex & NAtus & $\mathrm{DP}$ & silicone membrane & no \\
\hline Diamond Valve & Phoenix & Flow-regulating & diamond aperture & no \\
\hline Contour Flex & Radionic Medical P. & $\mathrm{DP}$ & silicone membrane & yes \\
\hline Sophy & Sophysa & ADJ & ball on spring & yes \\
\hline Sophy Mini & Sophysa & $\mathrm{DP}$ & ball on spring & yes \\
\hline Polaris & Sophysa & ADJ & ball on spring & Yes \\
\hline Synchrony & Ventura & $\mathrm{DP}$ & proximal slit & yes \\
\hline
\end{tabular}


techniques and armamentarium this scenario has completely changed with current mean perioperative and long-term mortality rate of $0,1 \%$ and $1,4 \%$, respectively. However, despite of the increasing variety of complex and expensive shunt devices becoming available for clinical use during the last decades, the treatment of hydrocephalus in adults and pediatric patients remains challenging, with approximately $40 \%$ of shunt-failure within two years of implantation and $98 \%$ failure after 10 years follow-up (7-9).

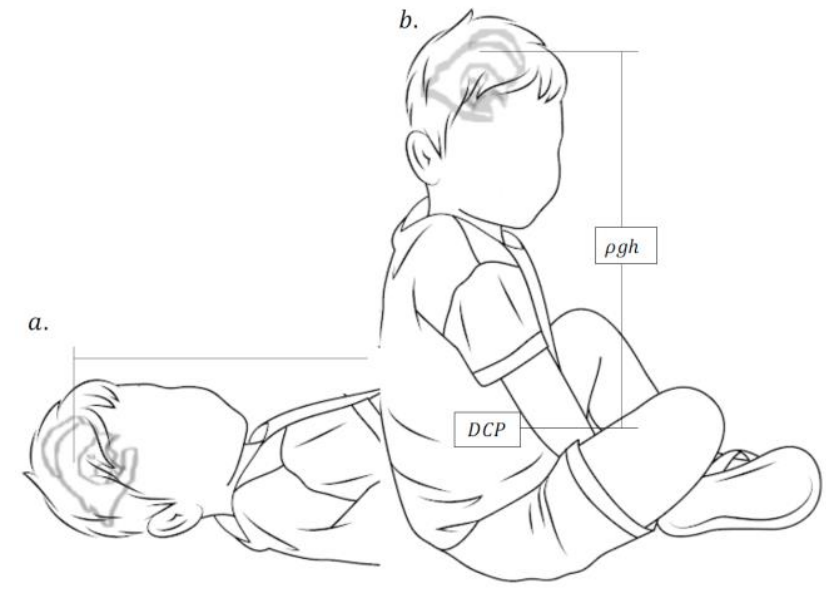

Figure 2 - Pressure gradient driving flow in a shunt system depending on body position. 2a. In the horizontal position flow depends on the intraventricular pressure, the opening pressure of the valve and on the distal cavity pressure; $2 \mathrm{~b}$. In the vertical (standing/sitting) position, intraventricular pressure is negative. In order to avoid overdrainage, the valve opening pressure should counteract the hydrostatic pressure. pgh: hydrostatic pressure, DCP: distal cavity pressure.

Since 1949, more than 200 different types of shunt devices have been described (10). Initial valves belonged to the group of first-generation differential pressure shunts and consisted of following working mechanisms: ball and cone, diaphragm, proximal slit and distal slit. Interestingly, the four technical principles for the construction of the first-generation valves were already established even before 1960 $(2,11,12)$. The second generation of shunts aimed to decrease the incidence of overdrainage. They included the first adjustable devices (Kuffer \& Strub, 1969 and Hakim, 1984), the flow-regulated systems (Orbis-Sigma, Sainte-Rose, 1987), and also introduced the concept of antisiphon (Portnoy \& Schulte 1973) and gravitational mechanisms (Hakim, 1975)(2). Figure 3 shows schematic representation of the working mechanism by shunt type.

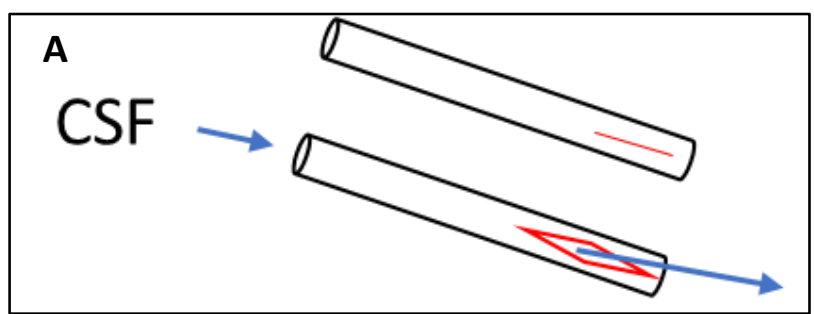

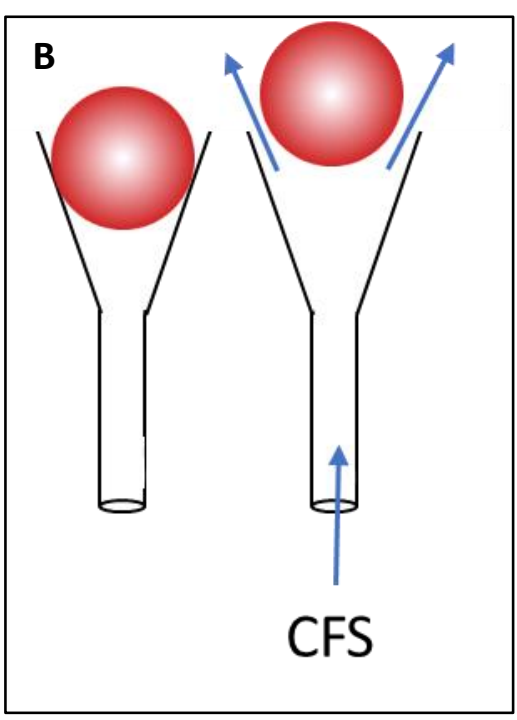

C
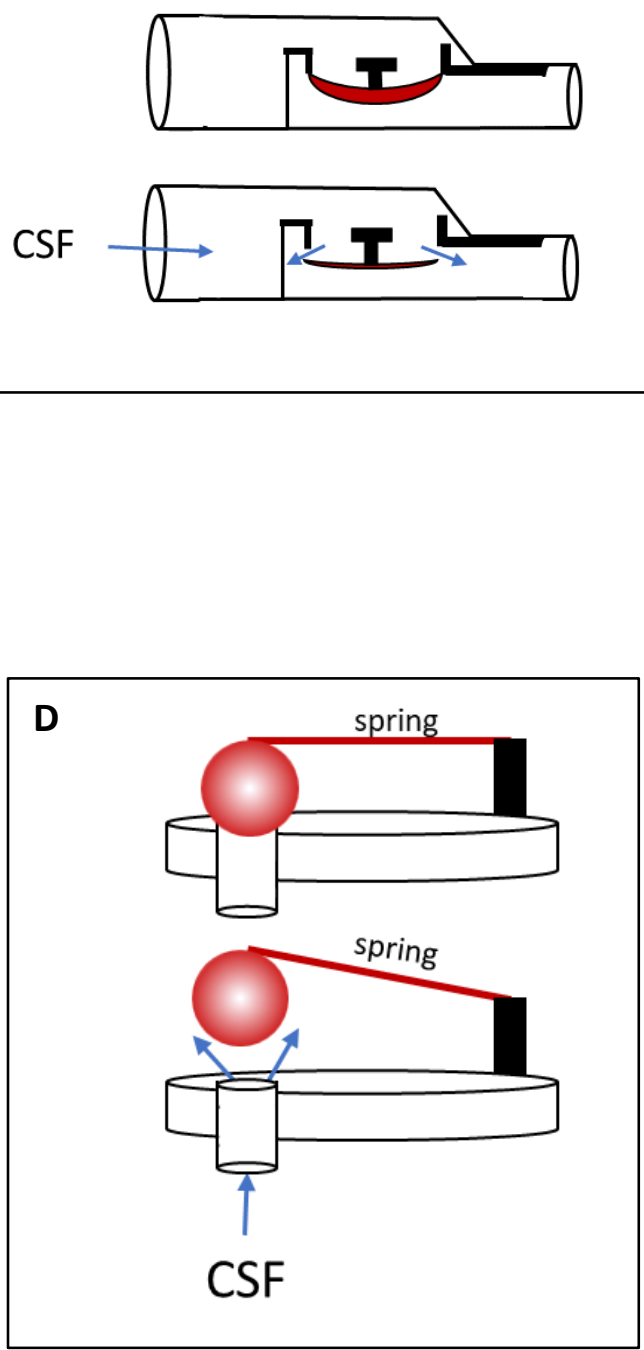

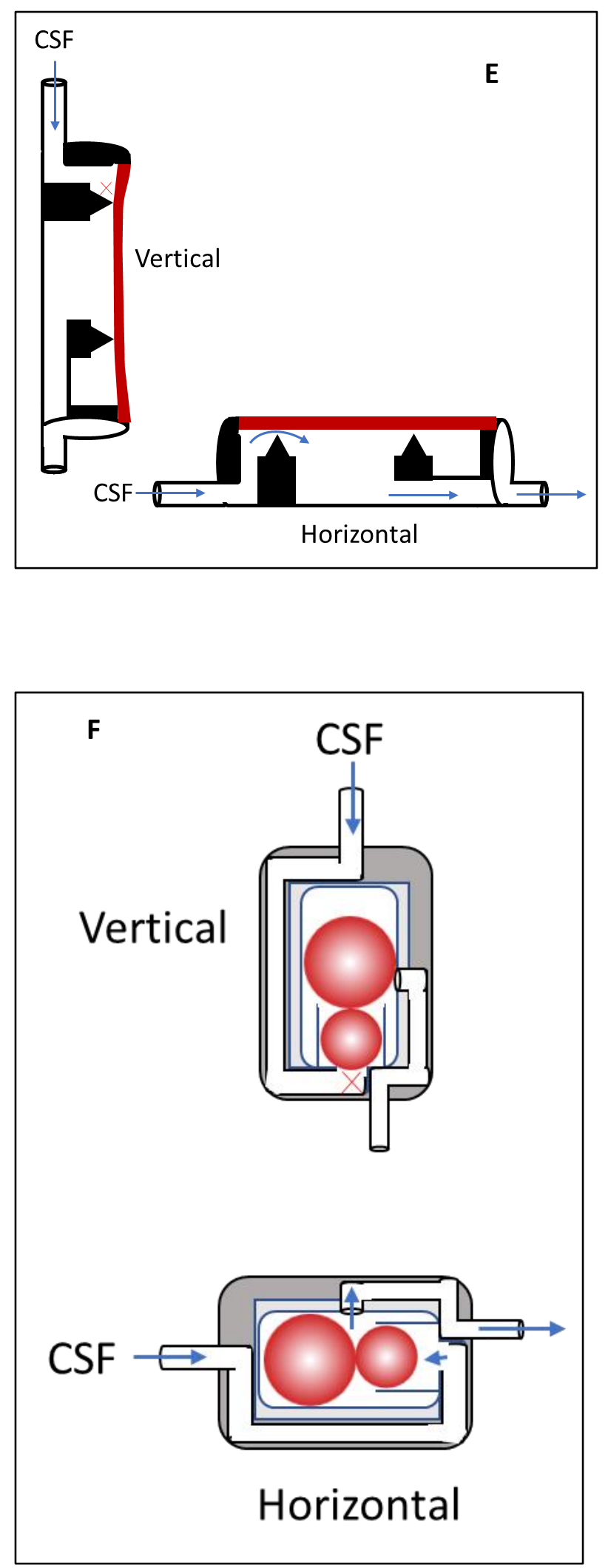

Figure 3- Schematic representation of the main shunt types. 3a. Slit valve; 3b. ball and cone; 3c. Diaphragm or silicon membrane; 3d. ball on spring; 3e. membrane anti-siphon device; 3f. gravitycontrolled balls anti-siphon device

\section{Adjustable shunts systems}

In 1973, Hakim et al introduced a shunt device that could be adjusted by a magnet over the skin (13). This was further developed until the adjustable MedosHakim became available and widely used from 1989. Currently, approximately eight types of adjustable designed are on the market (Table 1). The most common are the Codman Hakim Medos (spring-ball valve), the Medtronic Strata (ball and a magnetic rotor), the Sophy SM8 and Polaris (semicircular spring attached to the end of a pivoting bar) and the Miethke adjustable-gravitational devices. All these systems are theoretically MRI compatible, however some reports have shown irreparable damage of some devices, following exposure in an MRI environment (8). Therefore, it is advisable to routinely double-check the shunt settings after MRI imaging.

\section{Antisiphon and gravitational systems}

Siphoning effect is defined as the occurrence of overdrainage due to hydrostatic pressure as the patient stands. This can lead to serious deleterious effects, particularly to negative intracranial pressure, such as subdural hematomas, craniosynostosis and coma (14). Various antisiphon systems have been developed since the introduction of the Heyer-Schulte membrane device in 1973. The membranous compartments are sensitive to negative hydrostatic pressure, which closes the valve output as the patient assumes upright position (e.g. the Delta valve, built in the Medtronic Strata device). The gravitational devices were also designed to reduce overdrainage and consist of metal balls that gradually fall within a coneshaped seat as the patient moves from horizontal to vertical position, blocking the passage of CSF (e.g. the Miethke ProGAV). A well-known problem of gravitational valves is that an inadvertent displacement from its vertical alignment with the body results in changing the opening pressure and variable gravitational function $(8,10,15)$. Kiefer and Eymann (2009) reported a prospective analysis of 130 patients implanted with gravitational valves, with mean followup of $8.1 \pm 1.7$ years. All patients received adjustable or non-adjustable gravitational shunts (Miethke DualSwitch, Miethke Shunt-Assistante, adjustable Codman-Hakim and the Gravity-Assisted Valve GAV). They reported an overall $79 \%$ shunt-survival, 3\% infection rate and $5 \%$ overdrainage. The Siphonguard device (Codman Hakim) was also designed to reduce overdrainage through a flow regulating mechanism, however high flow can occur even in case of high resistance state. 


\section{Shunt sensors}

Currently, shunt performance is usually evaluated clinically and by imaging techniques, such as computed tomography and plain radiography. Shunt flow and CSF pressure can also be assessed by shunt tapping, however, these methods have clear limitations and expose patients to risks (i.e. ionizing radiation, infection, damage of the valve). Telemetric intracranial pressure monitoring seems to be a promising alternative to support diagnosis and fine adjustments in shunt-treated patients. In the last years, clinical and preclinical experience with shuntsensor devices have been reported $(8,16,17)$. Antes et al (2018) reported experience with the Miethke Sensor-reservoir in 25 patients presenting suboptimal improvement following shunting. The Shunt-reservoir consists of a device measuring $23.8 \mathrm{~mm}$ in diameter by $7.7 \mathrm{~mm}$ in height, which is attached to the proximal ventricular catheter at the level of the burr hole and distally to the rest of the shunt system. It has a built-in cell, which allows the non-invasive ICP monitoring in the presence of CSF flow (16). Measurements were taken with the patient standing, sitting and in the horizontal position. The authors showed that the telemetric ICP monitoring provided useful insight, allowing fine-tuning of the valve settings and improvement of clinical symptoms in 18 out of 25 patients $(72 \%)$. Interestingly, there was no correlation between optimal ICP and relief of symptoms or specific shunt settings (16). Although it may be an interesting strategy in selected difficult shunt cases, its large size, the impossibility to perform continuous ICP measurements and its prohibitive costs are considerable drawbacks of the Miethke Shunt-sensor.

\section{Scientific evidence and recommendations}

Recent studies evaluating the clinical impact of adjustable devices versus non-adjustable valves on outcome failed to provide evidence to recommend one or other in the treatment of pediatric hydrocephalus (Level II). Although higher rates of overdrainage have been observed with standard differential pressure valves, data available in the current medical literature is not sufficient to support any recommendation(18). Kestle et al (2000) reported a Class I, randomized controlled trial, comparing three types of valves: standard differential pressure, Delta (Medtronic) with antisiphon mechanism and Orbis-Sigma (Cordis) with variable resistance and flow-limiting feature (19). One hundred and seventy-seven patients were included across North America and Europe and followed-up for at least 12 months. The authors concluded that there was insufficient evidence to recommend the best shunt for initial treatment of hydrocephalus in children(19).

Warf et al (2005) published a prospective trial comparing the Codman-Hakim microprecision valve versus a standard low-cost differential pressure Chhabra device in 195 children. The end points of that study were shunt malfunction, migration, wound issues and death at 12 months follow-up. No statistically significant differences were observed $(p=$ $0.2)(20)$.

Several companies offer low-cost differential pressure shunt systems with antiphon mechanism. The mechanism is well-known and consists of one to three stainless steel weighting balls pressing on a sapphire ball with increasing pressure, from the horizontal to the vertical position, which increases the shunt opening pressure. Other more expensive gravitational valves work the same way. However, it has to be pointed out that some low-cost models do not dispose an anti-reflux valve, so that reflux towards the ventricles is likely to happen. Another caveat is the occurrence of overdrainage depending on patient's life activity, since some of these devices have low resistance to flow (15).

Beuriat et al (2017) reported a large retrospective study, which included 975 children submitted to shunt or ETV, with mean follow-up time of $11 \pm 7.4$ years. Six hundred ninety-five patients were submitted to shunt treatment, either using the Orbis-Sigma valve $(91.4 \%)$ or a standard differential pressure device (8.6\%).(21) The overall ETV survival analysis showed $76.9 \%$ at 1 year and $70 \%$ at 10 and 20 years. Regarding shunt treatment, the Orbis-Sigma showed better survival $(p=0.009)$ and less overdrainage $(p=$ 0.007 ), although valve obstruction occurred earlier than in the differential pressure group. This had been previously reported by other groups and is in line with its high-resistance valve concept (22).

A systematic review recently analyzed the impact of technological progress in reducing shunt failure. The authors pooled data from over 33,000 patients and concluded that pediatric shunt failure rates have not decreased over the last decades (23). One aspect that became evident was that early case series had higher number of late shunt failure (after 12 months follow-up), related to the higher number of ventriculoatrial shunts and migration of the distal catheter due to growth. Apart from that, no clear reduction of shunt complications (i.e. follow-up issues requiring revision or shunt replacement) was observed in the pediatric population (23). This may be explained by the fact that in most cases pediatric hydrocephalus can be adequately treated by any regular differential pressure shunt device. Advanced technology seems to play more important role in few selected cases.

Taken together, although one cannot neglect the contributions of technological advances to this field during the last decades, it seems that other important aspects might have been overlooked. First of all, more investments in research for a better understanding of the diverse underlying mechanisms of hydrocephalus are urgently needed. Secondly, the importance of teaching of proper surgical technique within neurosurgical residency programs must be reinforced 
for achievement of optimal results. Shunt surgery is often underestimated and regarded as a simple procedure by residents and neurosurgical staff, leading to avertible complications and unnecessary repeated surgeries. Simple problems such as kinking of the catheter, air bubbles within the system or even shortening of the distal catheter can all dramatically alter the dynamics of a shunt system, possibly favoring malfunction, obstruction or siphoning.

Finally, variables such as "shunt survival rate" and "shunt complication rate" often reported in shunt series must be interpreted with caution. Special attention must be given to the risk profile of a shunt device, which may be more relevant than simply the complication rate. Last but not the least, shunt survival depends not only on the underlying pathology, age and type of shunt device used, but also on the investigator's surveillance. Sometimes it may be challenging to differentiate between real shunt failure and functional failure of the device, so that high clinical suspicion and active vigilance is mandatory $(10,19,24)$.

\section{Conclusion}

Shunt technology has evolved during the last decades and continues to be intensively developed. Despite of the huge arsenal of different types of shunt systems available on the market, the treatment of hydrocephalus remains challenging. Knowing exactly how to choose the right shunt system in a specific patient still seems to be well beyond our current understanding about CSF dynamics in the CNS, especially in the brain already changed by hydrocephalus. Currently, scientific evidence does not support recommendations in favor of a specific type of shunt device for pediatric patients. Investment in research, education and training, as well as prospective randomized multicentric trials evaluating the role of specific valves in the treatment of individual group of hydrocephalic patients are urgently needed.

\section{References}

1. Nulsen FE, Spitz EB. Treatment of hydrocephalus by direct shunt from ventricle to jugular vain. Surg Forum. 1951;399-403.

2. Aschoff $A$, Kremer P, Hashemi B, Kunze S. The scientific history of hydrocephalus and its treatment. Neurosurg Rev. 1999 Oct;22(2-3):67-93; discussion 94-95.

3. Raffa G, Marseglia L, Gitto E, Germanò A. Antibiotic-impregnated catheters reduce ventriculoperitoneal shunt infection rate in high-risk newborns and infants. Childs Nerv Syst. 2015 Jul;31(7):1129-38.
4. Hakim S. Considerations on the physics of hydrocephalus and its treatment. Exp Eye Res. 1977;25 Suppl:391-9.

5. Penn RD, Linninger A. The physics of hydrocephalus. Pediatr Neurosurg. 2009;45(3):16174.

6. Riechert T, Umbach W. Die operative Behandlung des Hydrocephalus. In: Brandt P, Gerlach J, Gordh T, Irsigler FJ, Kleinsasser O, Mattos-Pimenta A, et al., editors. Klinik und Behandlung der Raumbeengenden Intrakraniellen Prozesse I [Internet]. Berlin, Heidelberg: Springer; 1960 [cited 2020 Apr 1]. p. 599672. (Handbuch der Neurochirurgie). Available from: https://doi.org/10.1007/978-3-642-48751-4_7

7. Drake JM, Kestle JR, Tuli S. Cerebrospinal fluid shunt technology. Clin Neurosurg. 2000;47:336-45.

8. Soler GJ, Bao M, Jaiswal D, Zaveri HP, DiLuna ML, Grant RA, et al. A Review of Cerebral Shunts, Current Technologies, and Future Endeavors. Yale J Biol Med. 2018;91(3):313-21.

9. Bondurant $\mathrm{CP}$, Jimenez DF. Epidemiology of cerebrospinal fluid shunting. Pediatr Neurosurg. 1995;23(5):254-8; discussion 259.

10. Aschoff A, Krämer P, Benesch C, Klank A. Shunttechnology and overdrainage--a critical review of hydrostatic, programmable and variable-resistancevalves and flow-reducing devices. Eur J Pediatr Surg. 1991 Dec;1 Suppl 1:49-50.

11. Chari A, Czosnyka M, Richards HK, Pickard JD, Czosnyka ZH. Hydrocephalus shunt technology: 20 years of experience from the Cambridge Shunt Evaluation Laboratory. J Neurosurg. 2014 Mar;120(3):697-707.

12. Oliveira MF de, Pereira RM, Pinto FG. Updating technology of shunt valves. MedicalExpress. 2014 May 5;1(4):166-9.

13. Hakim S. Hydraulic and mechanical mis-matching of valve shunts used in the treatment of hydrocephalus: the need for a servo-valve shunt. Dev Med Child Neurol. 1973 Oct;15(5):646-53.

14.Bober J, Rochlin J, Marneni S. Ventriculoperitoneal Shunt Complications In Children: An Evidence-Based Approach To Emergency Department Management. Pediatr Emerg Med Pract. 2016 Feb;13(2):1-22; quiz 22-3.

15. Czosnyka Z, Czosnyka M, Richards H, Pickard JD. Chhabra hydrocephalus shunt: lessons for gravitational valves. J Neurol Neurosurg Psychiatry. 1998 Sep;65(3):406-7.

16. Antes S, Stadie A, Müller S, Linsler S, Breuskin D, Oertel J. Intracranial Pressure-Guided Shunt Valve Adjustments with the Miethke Sensor Reservoir. World Neurosurg. 2018 Jan;109:e642-50.

17. Qin C, Olivencia-Yurvati AH, Williams AG, Eskildsen D, Mallet RT, Dasgupta PK. Inline flow sensor for ventriculoperitoneal shunts: Experimental evaluation in swine. Medical Engineering \& Physics. 2019 May 1;67:66-72.

18. Baird LC, Mazzola CA, Auguste KI, Klimo P, Flannery AM, Pediatric Hydrocephalus Systematic 
Review and Evidence-Based Guidelines Task Force. Pediatric hydrocephalus: systematic literature review and evidence-based guidelines. Part 5: Effect of valve type on cerebrospinal fluid shunt efficacy. J Neurosurg Pediatr. 2014 Nov;14 Suppl 1:35-43.

19. Kestle J, Drake J, Milner R, Sainte-Rose C, Cinalli $\mathrm{G}$, Boop $\mathrm{F}$, et al. Long-term follow-up data from the Shunt Design Trial. Pediatr Neurosurg. 2000 Nov;33(5):230-6.

20. Warf BC. Comparison of 1-year outcomes for the Chhabra and Codman-Hakim Micro Precision shunt systems in Uganda: a prospective study in 195 children. J Neurosurg. 2005 May;102(4 Suppl):35862.

21. Beuriat P-A, Puget S, Cinalli G, Blauwblomme T, Beccaria $\mathrm{K}$, Zerah $\mathrm{M}$, et al. Hydrocephalus treatment in children: long-term outcome in 975 consecutive patients. J Neurosurg Pediatr. 2017 Jul;20(1):10-8.
22. Smely C, Van Velthoven V. Comparative study of two customary cerebrospinal fluid shunting systems in early childhood hydrocephalus. Acta Neurochir (Wien). 1997;139(9):875-81; discussion 882.

23. Stein SC, Guo W. Have we made progress in preventing shunt failure? A critical analysis. J Neurosurg Pediatr. 2008 Jan;1(1):40-7.

24. Haberl EJ, Messing-Juenger M, Schuhmann M, Eymann R, Cedzich C, Fritsch MJ, et al. Experiences with a gravity-assisted valve in hydrocephalic children. Clinical article. J Neurosurg Pediatr. 2009 Sep;4(3):289-94. 